\title{
Certificates of optimality: the third way to biproportional apportionment
}

\author{
Paolo Serafini ${ }^{1}$, Bruno Simeone $e^{2}$ \\ ${ }^{1}$ University of Udine, Department of Mathematics and Computer Science, paolo.serafini@uniud.it \\ ${ }^{2}$ University La Sapienza, Rome, Department of Statistics, bruno.simeone@uniroma1.it
}

\begin{abstract}
The biproportional apportionment problem (BAP) must be faced in many proportional electoral systems where seats must be allocated to parties within regions. BAP is a non trivial optimization problem, and only sophisticated algorithms are currently available for solving it. The issue is: are they "writable" as an actual law? Citizens rightly demand simple, easy to understand, voting systems. The alternative, though, seems to have simple, but unsound electoral laws. We propose the following way out of this dilemma: leave to a mathematically sophisticated algorithm the task of producing an optimal apportionment, but attach to it a "certificate of optimality", that is, describe a simple procedure whereby anybody can check, through some elementary operations, that the seat allocation output by the algorithm is indeed an optimal apportionment. We discuss one such certificate, based on the Max Flow-Min Cut Theorem, relative to a parametric max flow method of ours for BAP.
\end{abstract}

\section{Introduction}

Electoral systems are usually quite complex and they are assembled out of many interacting components, which must be properly designed in order to achieve consistency and efficiency in the overall system. Sometimes it happens that designing a certain component is a highly nontrivial task. Mathematics can be of invaluable help in providing sound designs whose complexity escapes simple-minded procedures. As a matter of fact, when the latter are employed in order to solve an inherently difficult problem, severe inconsistencies and anomalies may occur. Therefore, there are actual cases where the use of mathematics is unavoidable.

However, it may happen that only mathematically sophisticated algorithms are available for solving a certain design problem. Are they "writable" as an actual law? Citizens rightly demand simple, easy to understand, voting systems (Principle 1 in the so-called "Erice Decalogue" [17]). Then the following dilemma may arise:

Which is better? To have simple, but unsound electoral laws, or sound, but complex ones?

In this paper, we propose the following way out:

Leave to a mathematically sophisticated algorithm the task of PRODUCING a sound solution, but attach to it a certificate of guarantee, that is, describe a simple procedure whereby ANYBODY CAN CHECK, through some elementary operations, that the solution output by the algorithm indeed satisfies all the requirements sought for. 
In the present paper we shall demonstrate this "third way" on a specific electoral design problem: biproportional apportionment. In many proportional electoral systems where several parties are in competition and the territory is subdivided into constituencies or regions, it is important to know how the seats are allocated to the parties within the regions. The problem of seat allocation to the parties within the regions is present in several countries, e.g. Germany, Italy, Mexico, Switzerland, Belgium, Denmark, Iceland, Faroe Islands, etc.

A well-known formulation of the above seat allocation problem (see, e.g., [2]) is as follows. Each region is granted a certain number of seats. This number is determined before the election on the basis of the region's population. Then each party is granted a certain number of seats at the national level. This number is determined after the election on the basis of the total number of votes obtained by the party. Then, given

(a) a matrix of votes obtained by each party in each region;

(b) the total number of seats apportioned to each region;

(c) the total number of seats granted to each party;

one has to find a matrix of seats allocated to each party within in each region. Such matrix must satisfy the following conditions:

(i) all of its entries must be nonnegative integers;

(ii) the sum of the seats assigned to all parties within a given region must be equal to the number of seats in the region;

(iii) the sum of the seats awarded to a given party in all regions must be equal to the total number of seats for the party.

(iv) if a party receives no vote in some region, it cannot receive seats in that region;

(v) the matrix of seats should be "as proportional as possible" to the matrix of votes.

A matrix satisfying conditions (i)-(iv) is called an apportionment. The requirement (v) needs a formal specification. The way this is done leads to different approaches in the literature. The above stated problem is called a biproportional apportionment problem (BAP).

Perhaps not suprinsingly, there are some electoral laws which fail to produce a correct procedure for solving BAP. For instance, Balinski and Ramírez [5] discovered a bug in the Mexican electoral law, which was later fixed. A similar bug turns out to occur in the current Italian law and this has not been fixed yet $[11,12,13,14]$.

On the other hand, mathematically sound procedures for BAP are indeed available. In two milestone papers [3, 4], Balinski and Demange characterized proportionality between real or integral matrices in terms of a set of axioms. It turns out that, for any given rounding rule, there is a unique apportionment satisfying the stated axioms. The algorithm for computing the apportionment calls for a sequence of scaling and rounding operations on the vote matrix, where rounding usually means to the lower, to the upper, or to the closest integer.

There are many sound procedures for BAP which have been later proposed. We mention the Discrete Alternate Scaling Method (DAS) [15, 9], the convex separable formulation of BAP by Gaffke and Pukelsheim [7, 8], the minimax parametric network flow method by Serafini and Simeone [16]. All these procedures share quite a degree of mathematical sophistication and so it is problematic to embody them in a real-life electoral law.

Formally the BAP problem to be solved is as follows. Before the elections the following data are given: a set $M$ of electoral regions, integer numbers $r_{i}, i \in M$, (seats assigned to region $i$ ), with $H:=\sum_{i \in M} r_{i}$ (house size), a set $N$ of parties. After elections integer numbers $p_{j}, j \in N$, and rational numbers $q_{i j}$, 
$i \in M, j \in N$, are computed from the votes. Let also $Z$ be the set of pairs $(i, j)$ such that party $j$ has received zero votes in region $i$. The numbers $p_{j}$ are the seats assigned to party $j$ at national level and the numbers $q_{i j}$, called target quotas, correspond to the ideal seats to be assigned in region $i$ to party $j$. We do not make any assumption on how the target quotas are computed, apart from setting $q_{i j}=0$ if $(i, j) \in Z$. Similarly we do not make any assumption on how the seats $p_{j}$ are computed, apart from the condition $\sum_{j} p_{j}=H$.

From these data one has to compute the actual seats $x_{i j}$ to be assigned in region $i$ to party $j$ such that

$$
\sum_{i \in M} x_{i j}=p_{j}, j \in N, \quad \sum_{j \in N} x_{i j}=r_{i}, i \in M, \quad x_{i j}=0,(i, j) \in Z .
$$

In this paper we consider the case where the criterion of choosing the seats (point (v) above) consists in minimizing an error measure with respect to the target quotas. In particular we assume that the computed seats are strongly optimal with respect to the the maximum absolute deviation from the target quotas, i.e.,

$$
\max _{i j}\left|x_{i j}-q_{i j}\right|
$$

Any apportionment satisfying (1) and minimizing (2) is optimal. An optimal solution is in addition strongly optimal if the following requirement holds (see also [16]). Let $x^{*}$ be a feasible apportionment. For a given region-party pair $(h, k)$, let $\tau_{h k}^{*}:=\left|q_{h k}-x_{h k}^{*}\right|$ be the error for this pair. Let $L(h, k):=\left\{(i, j) \neq(h, k): \tau_{i j}^{*} \leq \tau_{h k}^{*}\right\}$ be the set of pairs with error not larger than $\tau_{h k}^{*}$, and let $U(h, k):=$ $\left\{(i, j): \tau_{i j}^{*}>\tau_{h k}^{*}\right\}$ be the complement set. Then we say that the apportionment $x^{*}$ is strongly optimal if, for any pair $(h, k)$, there is no apportionment with error $\tau_{h k}<\tau_{h k}^{*}, \tau_{i j} \leq \tau_{h k}^{*}$ for $(i, j) \in L(h, k)$ and $\tau_{i j} \leq \tau_{i j}^{*}$ for $(i, j) \in U(h, k)$. Clearly strong optimality implies optimality (just consider the pair $(h, k)$ for which the maximum in (2) is attained).

The definition takes care of the fact that, while trying to improve the error for some pair $(h, k)$, it is not allowed to worsen those pairs which have a larger error than $(h, k)$. On the contrary, those pairs that have error less than or equal to $(h, k)$ are allowed to worsen the error up to the 'threshold' $\tau_{h k}^{*}$. Note also that if another pair exhibits the same error as $(h, k)$, the error for $(h, k)$ is allowed to decrease without any decrease of the error for the other pair.

As explained in [16], the problem of finding a unique strongly optimal apportionment can be modeled as a parametric capacitated transportation problem and efficiently computed by solving a sequence of Max Flow problems.

In this paper we are not concerned with algorithmic issues related to find such optimum. Rather we want to develop a "certificate of optimality" for that optimum. Even a layman with some very elementary computing skills can ascertain that the seat allocation produced by a not necessarily known algorithm is indeed a feasible apportionment, and that there is no feasible 'better' apportionment. Our certificate relies on the Max flow-Min cut Theorem of network flows, but this fact can be totally ignored by the layman. The certificate basically consists of two subsets, one of regions and the other one of parties. This two subsets are provided by the 'solver'. The layman has only to make simple computations by using the problem data and the two subsets together with their complements.

As a matter of fact we present two types of certificate. The first type, called global certificate, is related to the overall computation and guarantees that the apportionment is optimal. The second type, called individual certificate, consists of many certificates, one for each region-party pair, and guarantees that the solution is strongly optimal. The mathematical structure of these two types is the same.

Our paper is structured as follows. In Section 2 we provide an informal introduction to the notion of certificate of optimality under the form of a medieval tale. Section 3 lays out the theoretical foundations of our certificate and provides an example referring to the Italian political elections in 2008. The notion of certificate can be extended to all region-party pairs and this will be discussed in Section 4 . Section 5 finally, briefly offers some conclusions. 


\section{A medieval tale}

Virgil: It was brought to my knowledge that you have been appointed by the Township of Siena as the Official Verifier of their next elections.

Dante: You speak the truth, but I fear that also this time the electoral system adopted by the Township will produce inconsistencies and paradoxes.

Virgil: What happened?

Dante: Last time the Contrada (ward) Bruco got 4 seats, instead of the 5 granted to it by the Township Edict, while Contrada Oca got 2 seats instead of 1.

Virgil: I think I can do something for you. I know that one Pisan mathematician, named Leonardo Fibonacci, has left a booklet where he describes a correct method for your type of elections. Surely, his method will not give rise to the inconveniences you have complained about.

Dante: Looks interesting, but I am worried that the mathematical subtleties of his method will make the Elders of the Township quite suspicious and that eventually they will refrain from approving it.

Virgil: Don't worry. I happen to own a secret manuscript where Leonardo gives detailed explanations about how even a layman can check that the outcome of his method is correct.

\section{Three months later}

Dante: As instructed by you, I have handed you the table of votes obtained by the 6 parties in the 5 wards of Siena, together with the number of seats at stake in each ward (Table 1), and I got back from you a table of seats allocated to the parties in the wards according to Leonardo Fibonacci's method (Table 2).

Table 1:

\begin{tabular}{|l|r|r|r|r|r|r|r|}
\hline & Bottai & Cestari & Fabbri & Mugnai & Sediari & Vasari & Seats \\
\hline Aquila & 4960 & 4350 & 850 & 4970 & 4940 & 4930 & 5 \\
\hline Bruco & 2300 & 2900 & 4955 & 4965 & 4945 & 4935 & 5 \\
\hline Civetta & 0 & 0 & 0 & 4950 & 0 & 50 & 1 \\
\hline Drago & 0 & 0 & 0 & 2205 & 0 & 2795 & 1 \\
\hline Oca & 0 & 0 & 0 & 700 & 4300 & 0 & 1 \\
\hline
\end{tabular}

Table 2:

\begin{tabular}{|l|r|r|r|r|r|r|r|}
\hline & Bottai & Cestari & Fabbri & Mugnai & Sediari & Vasari & Seats \\
\hline Aquila & 1 & & & 2 & 1 & 1 & 5 \\
\hline Bruco & & 1 & 1 & 1 & 1 & 1 & 5 \\
\hline Civetta & & & & 1 & & & 1 \\
\hline Drago & & & & & & 1 & 1 \\
\hline Oca & & & & & 1 & & 1 \\
\hline Seats & 1 & 1 & 1 & 4 & 3 & 3 & 13 \\
\hline
\end{tabular}

Virgil: The last row of the table shows, for each party, the total number of seats attributed to the party in the whole township. It was obtained through a preliminary computation prior to the distribution of seats to parties within the wards.

Dante: No objection to the last row. In order to check it, I have completed my table (Table 3) with the total number of votes in each ward (row-sums) and for each party (column-sums). 
Table 3:

\begin{tabular}{|l|r|r|r|r|r|r|r|r|}
\hline & Bottai & Cestari & Fabbri & Mugnai & Sediari & Vasari & Total & Seats \\
\hline Aquila & 4960 & 4350 & 850 & 4970 & 4940 & 4930 & 25000 & 5 \\
\hline Bruco & 2300 & 2900 & 4955 & 4965 & 4945 & 4935 & 25000 & 5 \\
\hline Civetta & 0 & 0 & 0 & 4950 & 0 & 50 & 5000 & 1 \\
\hline Drago & 0 & 0 & 0 & 2205 & 0 & 2795 & 5000 & 1 \\
\hline Oca & 0 & 0 & 0 & 700 & 4300 & 0 & 5000 & 1 \\
\hline Total & 7260 & 7250 & 5805 & 17790 & 14185 & 12710 & 65000 & 13 \\
\hline
\end{tabular}

Then the Bottai party, with its 7260 votes, gets almost $11.17 \%$ of the votes, since $7260 / 65000=11.1692$; thus, it should get also $11.17 \%$ of the 13 seats, that is, 1.452 seats. Proceeding this way, I have computed the exact number of seats that each party deserves (Table 4)

Table 4:

\begin{tabular}{|l|r|r|r|r|r|r|r|}
\hline & Bottai & Cestari & Fabbri & Mugnai & Sediari & Vasari & Total \\
\hline Exact quotas & 1.452 & 1.45 & 1.161 & 3.558 & 2.837 & 2.542 & 13 \\
\hline
\end{tabular}

Virgil: The numbers you have computed are actually known as exact (or natural) quotas. Unfortunately, you cannot assign to a party a fractional number of seats. So, your exact quotas must be replaced by integer approximations.

Dante: That part is clear, and I see also how it is done in Leonardo's method. Each party is preliminarly assigned the integer part of its exact quota, for a total of 10 seats. The 3 remaining seats are assigned to the 3 parties with the largest decimal parts, that is, to the Sediari party with decimal part 0.837 , to the Mugnai party with decimal part 0.558 and to the Vasari party with decimal part 0.542 . In this way, one obtains the total number of seats attributed to the individual parties as shown in Table 2. It looks quite reasonable to me.

Virgil: What you have just described is known as the Largest Remainders Method.

Dante: Since the law of the town prescribes to deviate as little as possible from the quotas and the Largest Remainders Method does exactly that, I have tried to apply this method ward by ward. For example, in the Aquila ward the Bottai party gets $19.84 \%$ of the votes, since $4960 / 25000=0.1984$; so it should obtain $19.84 \%$ of the 5 available seats, that is, 0.992 seats. In this way I have computed the table of (wardwise) exact quotas (Table 5).

Table 5:

\begin{tabular}{|l|r|r|r|r|r|r|}
\hline & Bottai & Cestari & Fabbri & Mugnai & Sediari & Vasari \\
\hline Aquila & 0.992 & 0.870 & 0.170 & 0.994 & 0.988 & 0.986 \\
\hline Bruco & 0.460 & 0.580 & 0.991 & 0.993 & 0.989 & 0.987 \\
\hline Civetta & 0 & 0 & 0 & 0.990 & 0 & 0.010 \\
\hline Drago & 0 & 0 & 0 & 0.441 & 0 & 0.559 \\
\hline Oca & 0 & 0 & 0 & 0.140 & 0.860 & 0 \\
\hline
\end{tabular}

From that table I went on and computed, ward by ward, the number of seats to be assigned to the 6 parties by what you call the Largest Remainders method (Table 6).

But I was very disappointed, since some column sums were wrong: not always the sum of the seats given to a party in the different wards did match the total number of seats granted to that party. Actually, the 
Table 6:

\begin{tabular}{|l|r|r|r|r|r|r|r|}
\hline & Bottai & Cestari & Fabbri & Mugnai & Sediari & Vasari & Seats \\
\hline Aquila & 1 & 1 & & 1 & 1 & 1 & 5 \\
\hline Bruco & & 1 & 1 & 1 & 1 & 1 & 5 \\
\hline Civetta & & & & 1 & & & 1 \\
\hline Drago & & & & & & 1 & 1 \\
\hline Oca & & & & & 1 & & 1 \\
\hline Seats & 1 & 2 & 1 & 3 & 3 & 3 & 13 \\
\hline Granted Seats & 1 & 1 & 1 & 4 & 3 & 3 & 13 \\
\hline
\end{tabular}

Cestari party got one seat in excess, while the Mugnai party got one seat less. I can also add that I did again this computation proceeding party by party and assigning the seats to the wards. Yet, I could find the same type of wrong sums, this time on the rows.

On the other hand, I have checked that in the Leonardo's table both row- and column-sums are indeed correct. Namely, I have verified that such table meets the following requirements:

(i) every seats is an integer number greater than or equal to zero;

(ii) for each ward, the sum of the seats attributed to the 6 parties within that ward (row-sum) is equal to the number of seats at stake in that ward;

(iii) for each party, the sum of the seats attributed to the party in the 5 wards (column-sum) is equal to the number of seats granted to that party;

(iv) if a party gets no votes in a ward, it cannot receive any seat in that ward.

Virgil: For the sake of brevity, let us refer to a table of (integer) seats satisfying properties (i)- (iv) as an apportionment.

Dante: But I am puzzled by something strange. All exact quotas lying between 0 and 1, I would expect to see an apportionment where each entry is rounded either down to 0 or up to 1 . Instead the exact quota of the Mugnai party in the Aquila ward, i.e. 0.994, is approximated by 2 !

Virgil: There is no mistake. As a matter of fact, no matter how the exact quotas of Table 5 are rounded up or down, one can never get an apportionment satisfying all the requirements (i)-(iv).

Dante: How can you possibly make such a statement? The number of alternative ways to round up or down the 18 fractional entries of Table 5 is a huge number! How can you be sure that none of them gives rise to an apportionment?

Virgil: Be patient, and I will offer you an indisputable argument. But allow me to preliminarly make some simple considerations about approximating fractional numbers by integer ones. If you approximate the number 5 by 8 (which is 3 units above 5 ) or by 2 (which is 3 units below 5 ), in both cases the error you make amounts to 3 . Prescribing a maximum error of 3 with respect to 5 is tantamount to require that the approximating number lies in the interval between 2 and 8. Similarly, prescribing a maximum error of 4.25 w.r.t. 9.5 is equivalent to require that the approximating number lies in the interval $5.25-13.75$. If in addition you know that the approximating number is an integer, you can restrict the interval to 6 13. Are you following me?

Dante: Perfectly.

Virgil: Now, suppose I claim that the maximum error of Leonardo's apportionment (Table 2) w.r.t. the exact quotas (Table 5) is 1.006. How would you check my claim?

Dante: That's easy! Comparing Tables 2 and 5, I would compute a table of errors (Table 7), which shows that the maximum error is indeed 1.006 .

Virgil: For future purposes, let us double check on that. If the maximum error is 1.006, then in each cell of Table 2 the entry should lie in the interval between (exact quota -1.006 ) and (exact quota +1.006 ).

Notice that since in this case all fractional entries are smaller than 1.006, and since on the other hand 
Table 7:

\begin{tabular}{|l|r|r|r|r|r|r|}
\hline & Bottai & Cestari & Fabbri & Mugnai & Sediari & Vasari \\
\hline Aquila & 0.008 & 0.870 & 0.170 & 1.006 & 0.012 & 0.014 \\
\hline Bruco & 0.460 & 0.420 & 0.009 & 0.007 & 0.011 & 0.013 \\
\hline Civetta & 0 & 0 & 0 & 0.010 & 0 & 0.010 \\
\hline Drago & 0 & 0 & 0 & 0.441 & 0 & 0.441 \\
\hline Oca & 0 & 0 & 0 & 0.140 & 0.140 & 0 \\
\hline
\end{tabular}

seats are always nonnegative, we can safely set to 0 the lower extreme of the interval. Hence all entries of Table 2 should lie in the intervals indicated in Table 8 , and there should be at least one entry with error 1.006, making the entry coincide with its upper limit, and, since the seats must be integers, one can get the following tighter limits (Table 9).

Table 8:

\begin{tabular}{|l|r|r|r|r|r|r|}
\hline & Bottai & Cestari & Fabbri & Mugnai & Sediari & Vasari \\
\hline Aquila & $0-1.998$ & $0-1.876$ & $0-1.176$ & $0-2.000$ & $0-1.994$ & $0-1.990$ \\
\hline Bruco & $0-1.466$ & $0-1.586$ & $0-1.997$ & $0-1.999$ & $0-1.995$ & $0-1.993$ \\
\hline Civetta & & & & $0-1.996$ & & $0-1.016$ \\
\hline Drago & & & & $0-1.447$ & & $0-1.565$ \\
\hline Oca & & & & $0-1.146$ & $0-1.866$ & \\
\hline
\end{tabular}

Table 9:

\begin{tabular}{|l|c|c|c|c|c|c|}
\hline & Bottai & Cestari & Fabbri & Mugnai & Sediari & Vasari \\
\hline Aquila & $0-1$ & $0-1$ & $0-1$ & $0-2$ & $0-1$ & $0-1$ \\
\hline Bruco & $0-1$ & $0-1$ & $0-1$ & $0-1$ & $0-1$ & $0-1$ \\
\hline Civetta & & & & $0-1$ & & $0-1$ \\
\hline Drago & & & & $0-1$ & & $0-1$ \\
\hline Oca & & & & $0-1$ & $0-1$ & \\
\hline
\end{tabular}

Dante: Yes, I can see that the entries of Table 2 are within the intervals of Table 9, and that the number of seats attributed to the Mugnai party in the ward Aquila is equal to the upper limit 2, with an error 1.006 w.r.t. the exact quota 0.994. But couldn't there exist an apportionment whose maximum error is below 1.006 ?

Virgil: Let me convince you that no such apportionment may exist. From this it will ensue that Leonardo's apportionment is the best you can get, since it is impossible to decrease the maximum error of an apportionment. The crucial observation is the following. Look at the upper limit for Mugnai party in the Aquila ward in Table 9. Recall that this upper limit is $2=0.994+1.006$, where 0.994 is the exact quota. Now, suppose that the maximum error becomes smaller than 1.006. Then the upper limit becomes smaller than 2, and thus in Table 9 the upper limit of said cell becomes 1. All the other positive upper limits remain equal to 1 . Let me argue that there cannot be any apportionment satisfying all the limits in Table 9, with the upper limit 2 replaced by 1 . Notice that all entries of such an apportionment must be either 0 or 1 , since the only integers in the interval $0-1$ are 0 and 1 . By the way, this will provide a negative answer to your previous question on whether one can get an apportionment by suitably rounding 
down to 0 or up to 1 the fractional exact quotas in Table 5 .

Let me distinguish among the wards a set of wards of the Sun and a set of wards of the Moon. The Sun wards are Aquila and Bruco, the remaining wards are of the Moon. Similarly, let me call parties of the Sun the Bottai, Cestari, and Fabbri, and the remaining be the parties of the Moon. Now tell me: what is the largest number of seats within wards of the Sun that can be assigned to parties of the Moon?

Dante: Surely 6, because in either ward of the Sun one can assign at most 1 seat to each of the 3 Moon parties.

Virgil: Very good. Next: what is the least number of seats that can be attributed in Sun wards to Sun parties?

Dante: Obviously 4, since out of the 10 seats at stake in the 2 Sun wards at most 6 can go, as I said, to Moon parties. But now I see the contradiction which does not consent to it, since on the one hand the 3 Sun parties must collectively get at least 4 seats: on the other hand they are altogether granted only 3 seats (one seat each). Summing up, I agree on the fact that Leonardo's table provides a best apportionment, and I will recommend it to the Elders.

Virgil: I am quite confident that the Elders, though not profound in Mathematics as Leonardo, will be convinced by these common sense arguments. But now tell me: is it true that you are always eager of complete explanations?

Dante: Yes indeed, I am not satisfied until I have the whole picture in front of me.

Virgil: And is it true that you might be asked again to serve as the Official Verifier in some future election?

Dante: Yes, it might happen.

Virgil: Then there is one more thing to say.

Dante: What do you mean by this? Are you unfolding some hidden truth?

Virgil: No hidden truth. The only fact is that I applied Leonardo's arguments to this very special election in which the Bottai, Cestari and Fabbri parties received no votes in the Civetta, Drago and Oca wards. But suppose there were some votes and so the possible smallest and largest number of seats could be different from zero.

Dante: What consequence would this fact provoke?

Virgil: The consequence is that you cannot draw the conclusion that the Sun parties must receive 3 seats in the Sun wards. You should first consider the least number of seats that the Sun parties can receive in the Moon wards. This number would in turn give you the largest number of seats for the Sun parties in the Sun wards, and, if this number is smaller than the previous number which gave you the smallest number of seats, this clearly establishes a contradiction. Are you convinced by these arguments?

Dante: Yes, I am convinced.

Virgil: But my impression is that there is still something puzzling you. What are you actually thinking? Dante: Your explanations are very clear to me, but I am wondering whether it was only a lucky circumstance that you could find this division of parties and wards into Sun and Moon. I can see that by renaming Sun and Moon other groups of parties and other groups of wards there is no contradiction arising. How could I be sure that in another election a similar argument can be presented to the Elders? I realize that it requires the ability of a mathematician like Leonardo to find the right apportionment and the division of Sun-Moon parties and wards, but does this division exist in any case?

Virgil: Your intelligence has never disappointed me and I am glad that you have raised this question. In the secret manuscript by Leonardo it is also proved (and I am afraid it will take centuries before the mathematicians will rediscover this truth) that such division is always granted.

Dante: Now I am fully satisfied by your explanations and I am very grateful to you. Once more you are my real Guide and Master.

One month later:

Virgil: Have you been able to convince the Elders of the correct seat assignment? 
Dante: Yes, everything went smoothly and the Elders praised a lot my arguments. Unfortunately after one week the Cestari party started complaining about their seat assignment. They were granted only one seat which, according to Leonardo's table, was given in the Bruco ward, but due to some obscure reasons they prefer this seat in the Aquila ward. They argue that having a quota of 0.87 in the Aquila ward and 0.58 in the Bruco ward, their seat should be assigned in the Aquila ward. The question is on the hands of the Elders which are about to summon me again to settle the question.

Virgil: So, how do you think of settling the matter?

Dante: I do not know really. I thought that I could use the same arguments and so I prepared a new table of limits, like the one we did before (Table 9). This time however, I thought I had to keep the high error for the Mugnai party in the Aquila ward and leave the interval as 0-2. Indeed we already proved that it was not possible to narrow this interval to $0-1$.

Virgil: Yes, you did correctly.

Dante: Then in the new table I brought the interval for the Cestari party in the Aquila wards to 1-1, exactly to meet their request. But at the same time I did not feel like allowing an error of 0.992 to the Bottai party in the Aquila ward. Since 0.992 is larger than 0.87 it seems unfair to lower one error at the expense of an even larger error for others. Hence the interval for the Bottai party in the Aquila ward is $1-1$.

Virgil: This is perfectly reasonable.

Dante: And the same is true for all other ward-party pairs with error larger than 0.87 . So this is the table (Table 10) I have obtained:

Table 10:

\begin{tabular}{|l|c|c|c|c|c|c|}
\hline & Bottai & Cestari & Fabbri & Mugnai & Sediari & Vasari \\
\hline Aquila & $1-1$ & $1-1$ & $0-1$ & $0-2$ & $1-1$ & $1-1$ \\
\hline Bruco & $0-1$ & $0-1$ & $1-1$ & $1-1$ & $1-1$ & $1-1$ \\
\hline Civetta & & & & $1-1$ & & $0-0$ \\
\hline Drago & & & & $0-1$ & & $0-1$ \\
\hline Oca & & & & $0-1$ & $0-1$ & \\
\hline
\end{tabular}

Virgil: It looks correct.

Dante: But now you can see! This time I do no find any contradiction in computing as before the possible seats. The Aquila and Bruco wards are entitled at least $10-7=3$ seats, and this does not lead to a contradiction.

Virgil: Of course you cannot find the contradiction in this way. You need a different group of Sun and Moon parties and wards. My suggestion is that you try with only Bruco as a Sun ward and the Bottai and Cestari as Sun parties.

Dante: So let me try this way. In the Bruco ward 5 seats are at stake. At most 4 seats can be assigned to the Moon parties (Fabbri, Mugnai, Sediari and Vasari) and therefore at least one seat must be assigned between the Bottai and Cestari parties. And then ... it looks there is no contradiction because they have to receive two seats after all.

Virgil: Remember what I have told you last time. There is another symmetrical consideration to take care of.

Dante: Sure, now I remember. In the Moon wards (Aquila, Civetta, Drago, Oca) at least 2 seats must be assigned to the Sun parties so the Sun parties can receive in the Sun wards at most $2-2=0$ seats. Here there is the contradiction! Now I can safely report to the Elders that the Cestari request cannot be fulfilled. 


\section{A global certificate}

Let us call 'Solver' the person who actually solves the biproportional apportionment problem described in Section 1 and 'Verifier' the person who has to check the validity of the solution provided by the Solver. For the Verifier to check the validity, the Solver has to provide also a certificate, which consists in a subset $I$ of regions (let $\bar{I}$ be the complementary subset) and a subset $J$ of parties (let $\bar{J}$ be the complementary subset). These subsets, together with the given solution $x_{i j}^{*}$ and corresponding absolute error $\tau^{*}$, enable the Verifier to check the validity of the solution through some elementary steps. Indeed the only mathematical "skills" required to the Verifier are:

(1) to be able to perform ordinary sums and subtractions;

(2) to be able to compare two numbers to tell whether one of them is smaller than or equal to the other;

(3) to know the meaning of $\lfloor a\rfloor,\lceil a\rceil$ and $a^{+}$for a given (rational) number $a$ and to be convinced of the following simple facts:

(a) for any number $a,\lfloor a\rfloor \leq a$ and $\lceil a\rceil \geq a$,

(b) if $x$ is an integer, then $a \leq x \leq b$ if and only if $\lceil a\rceil \leq x \leq\lfloor b\rfloor$,

if $x$ is a nonnegative integer, then $a \leq x \leq b$ if and only if

$\lceil a\rceil^{+} \leq x \leq\lfloor b\rfloor$,

(c) if $a$ and $b$ are numbers such that $a \leq b$, then

$\lfloor a\rfloor \leq\lfloor b\rfloor$ and $\lceil a\rceil \leq\lceil b\rceil$ (it is implied by (a) and (b),

(d) if $b$ is integer and $b^{\prime}<b$, then $\left\lfloor b^{\prime}\right\rfloor \leq b-1$,

if $a$ is an integer and $a^{\prime}>a$, then $\left\lceil a^{\prime}\right\rceil \geq a+1$.

Then the Verifier, equipped with these basic skills, should first

- check that $\sum_{i \in M} x_{i j}^{*}=p_{j}, \sum_{j \in N} x_{i j}^{*}=r_{i}, x_{i j}^{*}=0$ for $(i, j) \in Z$ and $\tau^{*}=\min _{i j}\left|x_{i j}^{*}-q_{i j}\right|$, that is, $x^{*}$ is a feasible apportionment and its error is the one claimed (skills 1 and 2).

Next the Verifier, in order to be convinced that there can be no apportionment with error $\tau<\tau^{*}$, should first:

- compute $\alpha_{i j}:=\left\lceil q_{i j}-\tau\right\rceil^{+}$, with $i \in \bar{I}$ and $j \in J, \beta_{i j}:=\left\lfloor q_{i j}+\tau\right\rfloor$, with $i \in I$ and $j \in \bar{J}$, if $(i, j) \notin Z$ $\left(\alpha_{i j}=\beta_{i j}=0\right.$ if $\left.(i, j) \in Z\right)$ (skill 1$)$;

- compute $\bar{\alpha}:=\sum_{i \in \bar{I}} \sum_{j \in J} \alpha_{i j}$ and $\bar{\beta}:=\sum_{i \in I} \sum_{j \in \bar{J}} \beta_{i j}$ (skill 1).

and then reason as follows:

- if the maximum absolute error with respect to the target quotas over all seat assignments is $\tau$, then every seat assignment $x_{i j}$ must lie in the interval $\left[q_{i j}-\tau, q_{i j}+\tau\right]$ (skill 2) and this interval, due the integrality and nonnegativity of $x_{i j}$ can be equivalently restricted to $\left[\left\lceil q_{i j}-\tau\right\rceil^{+},\left\lfloor q_{i j}+\tau\right\rfloor\right]=\left[\alpha_{i j}, \beta_{i j}\right]$ (skill 3(b));

- if an apportionment $x$ with maximum absolute error $\tau$ is required, then the largest number of seats which can be assigned to parties in $\bar{J}$ in regions in $I$ is $\bar{\beta}$ and consequently the smallest number of seats which can be assigned to parties in $J$ in regions in $I$ is $\sum_{i \in I} r_{i}-\bar{\beta}$ (refer to Fig. 1(a) which represents the table of seats; the rows are the regions and the columns are the parties; in the figure $R:=\sum_{i \in I} r_{i}$ ) (skills 1 and 2);

- similarly, if an apportionment $x$ with maximum absolute error $\tau$ is required, then the smallest number of seats which can be assigned to parties in $J$ in regions in $\bar{I}$ is $\bar{\alpha}$ and consequently the largest number of seats which can be assigned to parties in $J$ in regions in $I$ is $\sum_{j \in J} p_{j}-\bar{\alpha}$ (refer to Fig. 1(b) where $\left.P:=\sum_{j \in J} p_{j}\right)($ skills 1 and 2$)$;

- finally, if

$$
\sum_{j \in J} p_{j}-\bar{\alpha}<\sum_{i \in I} r_{i}-\bar{\beta}
$$




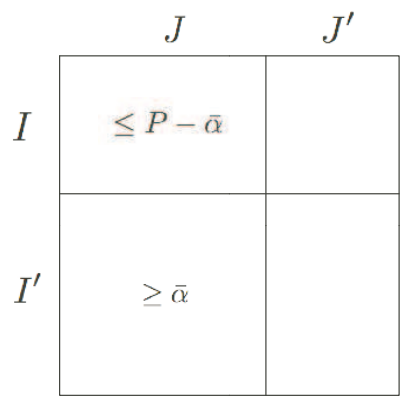

(a)

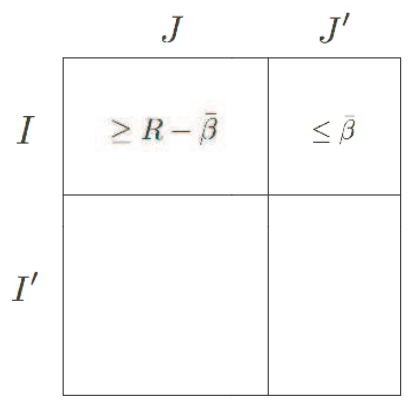

(b)

Figure 1:

that is, the largest number of seats is less than the smallest number of seats, then it is impossible to have an apportionment with error $\tau$ (skill 2). It is also impossible to have an apportionment with any smaller error because $\bar{\beta}$ decreases and $\bar{\alpha}$ increases if $\tau$ decreases (skill 3(c)).

In order to be convinced of the validity of the previous statements, only elementary reasoning is required. Note that the mathematical techniques to get the optimal solution can be totally ignored by the Verifier.

The existence of subsets $I$ and $J$ for which $\sum_{j \in J} p_{j}-\bar{\alpha}<\sum_{i \in I} r_{i}-\bar{\beta}$ if $\tau<\tau^{*}$ is established by Gale's theorem $[1,10]$ which states that there exists a feasible flow in a network if and only if for each subset of nodes the net flow out of the subset must be not larger than the cut capacity induced by the node subset. Since finding a feasible solution with maximum error bounded by $\tau$ is equivalent to finding a feasible flow on the bipartite network $(M, N ; E)$ (where $E=\{(i, j): i \in M, j \in N\}$ ) for the problem:

$$
\begin{array}{rlrl}
\left\lceil q_{i j}-\tau\right\rceil^{+} \leq x_{i j} \leq\left\lfloor q_{i j}+\tau\right\rfloor & & (i, j) \in E \backslash Z \\
x_{i j} & =0 & & (i, j) \in Z \\
\sum_{i \in M} x_{i j} & =p_{j} & & j \in N \\
\sum_{j \in N} x_{i j} & =r_{i} & & i \in M
\end{array}
$$

where $\lceil a\rceil^{+}=\max \{0,\lceil a\rceil\}$. with $M$ a set of sources $r_{i}$ and $N$ a set of $\operatorname{sinks} p_{j}$, then $\sum_{i \in I} r_{i}-\sum_{j \in J} p_{j}$ is the net flow out of the node subset $I \cup J$ and $\bar{\beta}-\bar{\alpha}$ is the cut capacity. The computation of the subsets is carried out by standard max flow techniques.

The above procedure "challenges" the Verifier to check the certificate for any trial $\tau<\tau^{*}$. However, the Verifier might be still in doubt whether there is a "feasible" error between the largest $\tau$ ever checked and $\tau^{*}$. The following additional argument shows that it is enough to ascertain the nonexistence of a feasible apportionment for a single set of bounds.

First the Verifier has to check the feasibility of the given apportionment and that its error is $\tau^{*}$. During this computation he has also to list the pairs of indices $(h, k)$, such that $\tau^{*}=\left|x_{h k}^{*}-q_{h k}\right|$. Let us call these pairs critical. For any critical pair we have

$$
\begin{array}{lll}
\text { either } & i) & x_{h k}^{*}=q_{h k}-\tau^{*} \\
\text { or } & \text { ii) } & x_{h k}^{*}=q_{h k}+\tau^{*}
\end{array}
$$

We may say that in case $i)$ the critical pair $(h, k)$ is a lower pair and in case ii) it is an upper pair. In other words $q_{h k}-\tau^{*}$ is integral for a lower pair and $q_{h k}+\tau^{*}$ is integral for an upper pair. 
In the medieval tale of Sec. 2, there is only one critical pair $(h, k)$, namely, the upper pair (Aquila, Mugnai), with $2=x_{h k}^{*}=q_{h k}+\tau^{*}=0.994+1.006$. When the maximum error $\tau$ becomes smaller than $\tau^{*}$, $q_{h k}+\tau$ becomes strictly smaller than 2 . Therefore, the upper bound on the (integer) number of seats $x_{h k}$ cannot be larger than 1. But already the upper bound 1, the other bounds being unchanged, makes the BAP infeasible.

Now the Verifier has to notice that, among the critical pairs, there is one such that, if it is a lower pair then $h \in \bar{I}$ and $k \in J$, whereas if it is an upper pair then $h \in I$ and $k \in \bar{J}$. Let us call any such critical pair a blocking pair. The Verifier does not need to be convinced that a blocking pair does indeed exist. He has only to scan all pairs $(i, j)$ until he finds a blocking pair.

Then the Verifier is asked to compute the $\alpha_{i j}\left(\tau^{*}\right)$ and $\beta_{i j}\left(\tau^{*}\right)$ values w.r.t. the error $\tau^{*}$, for the same sets $I$ and $J$. Here we have explicitly denoted the dependance of these values on $\tau$. Now the Verifier is invited to think what happens to $\alpha_{i j}(\tau)$ and $\beta_{i j}(\tau)$ for an error $\tau<\tau^{*}$. Since $\alpha_{i j}(\tau)=\left\lceil q_{i j}-\tau\right\rceil^{+}$and $q_{i j}-\tau>q_{i j}-\tau^{*}$, then by skill 3(c) we have $\alpha_{i j}(\tau) \geq \alpha_{i j}\left(\tau^{*}\right)$. Similarly, from $\beta_{i j}(\tau)=\left\lfloor q_{i j}+\tau\right\rfloor$ and $q_{i j}+\tau<q_{i j}+\tau^{*}$, again by skill $3(\mathrm{c})$ we have $\beta_{i j}(\tau) \leq \beta_{i j}\left(\tau^{*}\right)$. These inequalities are clearly valid for all pairs $(i, j)$.

The crucial point concerns the blocking pair. Here the Verifier needs the skill $3(\mathrm{~d})$. Indeed if the blocking pair is lower, $q_{h k}-\tau^{*}$ is integral and nonnegative (it is just a seat number and cannot be negative). Hence for any $\tau<\tau^{*},\left\lceil q_{i j}-\tau\right\rceil \geq\left\lceil q_{i j}-\tau^{*}\right\rceil+1$, i.e. $\alpha_{h k}(\tau) \geq \alpha_{h k}\left(\tau^{*}\right)+1$. Then the Verifier acknowledges that he has to use at least the lower bound $\alpha_{h k}\left(\tau^{*}\right)+1$ for the lower blocking pair $(h, k)$ if he wants to consider errors $\tau<\tau^{*}$.

If the blocking pair is upper we have that $q_{h k}+\tau^{*}$ is integral. Hence for any $\tau<\tau^{*},\left\lfloor q_{i j}+\tau\right\rfloor \leq$ $\left\lfloor q_{i j}+\tau^{*}\right\rfloor-1$, i.e. $\beta_{h k}(\tau) \leq \beta_{h k}\left(\tau^{*}\right)-1$. Then the Verifier acknowledges that he has to use at most the upper bound $\beta_{h k}\left(\tau^{*}\right)-1$ for the upper blocking pair $(h, k)$ if he wants to consider errors $\tau<\tau^{*}$.

Now the Verifier, equipped with the values $\alpha_{i j}\left(\tau^{*}\right):=\left\lceil q_{i j}-\tau^{*}\right\rceil^{+}$, with $i \in \bar{I}$ and $j \in J,(i, j)$ not blocking, $\beta_{i j}\left(\tau^{*}\right):=\left\lfloor q_{i j}+\tau^{*}\right\rfloor$, with $i \in I$ and $j \in \bar{J},(i, j)$ not blocking, $\alpha_{i j}=\beta_{i j}=0$ if $(i, j) \in Z$, and either $\alpha_{h k}\left(\tau^{*}\right)+1$ for the lower blocking pair $(h, k)$ or $\beta_{h k}\left(\tau^{*}\right)-1$ for the upper blocking pair $(h, k)$, proceeds with the previous computations of $\bar{\alpha}$ and $\bar{\beta}$. He will eventually find the contradiction

$$
\sum_{j \in J} p_{j}-\bar{\alpha}<\sum_{i \in I} r_{i}-\bar{\beta}
$$

If the Verifier had used $\alpha_{i j}(\tau)$ and $\beta_{i j}(\tau)$ (instead of $\alpha_{i j}\left(\tau^{*}\right), \beta_{i j}\left(\tau^{*}\right)$ and $\alpha_{h k}\left(\tau^{*}\right)+1$ or $\left.\beta_{h k}\left(\tau^{*}\right)-1\right)$ the contradiction would arise anyway because of the previously found inequalities $\alpha_{i j}(\tau) \geq \alpha_{i j}\left(\tau^{*}\right), \beta_{i j}(\tau) \leq$ $\beta_{i j}\left(\tau^{*}\right), \alpha_{h k}(\tau) \geq \alpha_{h k}\left(\tau^{*}\right)+1, \beta_{h k}(\tau) \leq \beta_{h k}\left(\tau^{*}\right)-1$. Notice that these inequalities hold as equalities for any $\tau$ in a suitable left neighborhood of $\tau^{*}$; but the actual value of $\tau$ is irrelevant for the Verifier.

Sometimes the impossibility of an apportionment for a given error is due to a simple fact, which is almost self-evident and does not require a special mathematical knowledge. Although this kind of impossibility is only a particular case of the previous general case, it is however more convenient to deal with it separately, just because it is a certificate that does not need many explanations. Indeed if for a given party $j$ it happens that

$$
\text { either } \quad \sum_{i \in M}\left\lfloor q_{i j}+\tau\right\rfloor=\sum_{i \in M} \beta_{i j}<p_{j} \quad \text { or } \quad \sum_{i \in M}\left\lceil q_{i j}-\tau\right\rceil^{+}=\sum_{i \in M} \alpha_{i j}>p_{j}
$$

then there is clearly no way of assigning seats in the regions for party $j$ and reaching the prescribed national number of seats $p_{j}$. Similarly, if for a given region $i$ it happens that

$$
\text { either } \quad \sum_{j \in N}\left\lfloor q_{i j}+\tau\right\rfloor=\sum_{j \in N} \beta_{i j}<r_{i} \quad \text { or } \quad \sum_{j \in N}\left\lceil q_{i j}-\tau\right\rceil^{+}=\sum_{j \in N} \alpha_{i j}>r_{i}
$$

then again there is no way of assigning seats in region $i$ to the parties and reaching the prescribed regional number of seats $r_{i}$. We leave the reader to check that the four cases correspond to the following subset 
specifications:

$$
\begin{array}{lll}
p_{j}>\sum_{i \in M} \beta_{i j} & \Longrightarrow I=M, & J=N \backslash\{j\}, \\
p_{j}<\sum_{i \in M} \alpha_{i j} & \Longrightarrow I=\emptyset, & J=\{j\}, \\
r_{i}>\sum_{j \in N} \beta_{i j} \quad \Longrightarrow \quad I=\{i\}, & J=\emptyset, \\
r_{i}<\sum_{j \in N} \alpha_{i j} \quad \Longrightarrow \quad I=M \backslash\{i\}, & J=N .
\end{array}
$$

We apply these concepts to the results of the Italian political election (April 13-14, 2008) for the Chamber of Deputies. The target quotas in the 26 regions, already modified to take into account the majority bonus to the winning coalition (PDL, LN, MPA), are reported in Table 11. The regions are listed by rows and the parties by columns. The party acronyms are: PDL - Popolo delle libertà, LN - Lega Nord, MPA - Movimento per le autonomie, PD - Partito democratico, IDV - Italia dei Valori, SVP - Südtiroler VolksPartei, UDC - Unione di Centro.

The seats assigned by the law to the regions, according to the population, before the elections are

$$
r=\{24,22,40,43,15,10,29,20,13,17,43,38,
$$

$$
9,16,40,15,14,3,33,29,44,6,22,26,28,18\}
$$

and the seats granted to the parties at the national level (according to the Largest Remainder Rule) are

$$
p=p^{N}=\{272,60,8,211,28,2,36\}
$$

As already pointed out in $[11,12,13,14,16]$ the final seats assigned in each region after the election are different from the ones assigned by the law before the election. But this is only due to a flaw in the procedure of the Italian law. In our computation we have used the original $r$ values. In the table the quotas have been rounded to three decimal digits.

We report in Table 12 the strongly optimal apportionment computed via the algorithm in [16]. Indeed we claim that it is optimal and we provide the following certificate to support our claim:

$I=\{$ Piemonte 1, Lombardia 2, Lombardia 3, Trentino-Alto Adige,

Friuli-Venezia Giulia, Liguria, Toscana, Umbria, Lazio 2, Abruzzo, Molise,

Campania 1, Campania 2, Puglia, Basilicata, Calabria, Sicilia 1\}

$$
J=\{\mathrm{PDL}, \mathrm{MPA}, \mathrm{PD}, \mathrm{IDV}, \mathrm{SVP}, \mathrm{UDC}\}
$$

The Verifier can compute the maximum error which is 0.75372 for the lower pair (Veneto 1, UDC) (1 seat against a target quota of 1.75372). Then, from the subsets $I$ and $J$ the $\alpha_{i j}$ and $\beta_{i j}$ values can be computed by trying for instance an error $\tau=0.7537$, as in Table 13, with $\alpha_{i j}, i \notin I, j \in J$ on the left and $\beta_{i j}, i \in I$, $j \notin J$ on the right.

From the table the Verifier can deduce that the largest number of seats in $(I, J)$ is given by 557 (total seats for parties in $J)-223\left(=\sum \alpha_{i j}=\bar{\alpha}\right)=334$, and also that the smallest number of seats in $(I, J)$ is given by 361 (total seats in regions in $I)-26\left(=\sum \beta_{i j}=\bar{\beta}\right)=335$. Therefore it is impossible to have a solution with error $\tau=0.7537$.

For the stronger form of verification, the Verifier needs to compute the $\alpha_{i j}\left(\tau^{*}\right)$ and $\beta_{i j}\left(\tau^{*}\right)$ values. These values are the same as in Table 13 with the exception of the lower blocking pair $(h, k)=$ (Veneto 1 , UDC) for which $\alpha_{h k}\left(\tau^{*}\right)=1$ (instead of 2). Clearly, using in the computation of $\bar{\alpha}, \alpha_{h k}\left(\tau^{*}\right)+1=2$ produces the same contradiction. 


\section{Strongly optimal solutions and individual certificates}

In general optimal solutions of problems minimizing an $L_{\infty}$-norm, like (1), are not unique. If the $L_{\infty}$-norm corresponds to a multi-objective minimization of all components of a multi-dimensional quantity (like to values $\left|q_{i j}-x_{i j}\right|$ ), then it may happen that some optima are 'worse' than other optima. For this reason we have introduced the concept of strong optimality. Strong optima cannot be 'worse' than other optima and the definition provided in the Introduction formalizes this concept.

Clearly a strongly optimal solution is robust with respect to any criticism and complaint parties might raise in order to gain more seats for themselves or to reduce seats for the other parties. More importantly, also strongly optimal solutions can be attached a certificate of optimality which can be checked against any complaint. We call this an individual certificate, since it is provided for each region-party pair. In contrast we may call global the certificate introduced in the previous section.

First note that no pair can have error less than

$$
\bar{\tau}_{i j}:=\min \left\{q_{i j}-\left\lfloor q_{i j}\right\rfloor ;\left\lceil q_{i j}\right\rceil-q_{i j}\right\} .
$$

So, if $\left|q_{i j}-x_{i j}^{*}\right|=\bar{\tau}_{i j}$, or, more simply, if the error is no larger than one half, the apportionment cannot be improved for the pair $(i, j)$. Since this is obvious we need a certificate only for those pairs exhibiting an error larger than one half.

The individual certificate has the same features as the global certificate, that is, it specifies two subsets of regions and parties. It only differs in the way the values $\alpha_{i j}$ and $\beta_{i j}$ are computed. According to the definition of strong optimality, when we consider the pair $(h, k)$, we set the error values $\tau_{i j}$ as

$$
\tau_{h k}<\tau_{h k}^{*}, \quad \tau_{i j}:=\tau_{h k}^{*}, \quad(i, j) \in L(h, k), \quad \tau_{i j}:=\tau_{i j}^{*} \quad(i, j) \in U(h, k)
$$

from which we compute

$$
\alpha_{i j}:=\left\lceil q_{i j}-\tau_{i j}\right\rceil^{+}, \quad \beta_{i j}:=\left\lfloor q_{i j}+\tau_{i j}\right\rfloor
$$

Since by definition there is no apportionment with this error, in the network flow problem (3) with capacities computed from $\tau_{i j}$ there must exist, by Gale's theorem, a subset of nodes whose cut capacity is not large enough to accomodate the net flow out of the subset. This subset of nodes is clearly the certificate.

In the final part of the medieval tale the considerations to show that the Cestari party request cannot be fulfilled are indeed an individual certificate for the pair (Aquila, Cestari). As a second example reconsider the solution for the Italian election 2008 reported in Table 12. Although acknowledging that the best maximum error is 0.75372 , the MPA party, having a quota of 1.626076 , could claim 2 seats instead of 1 in the Sicily 1 region. To prove that MPA cannot receive 2 seats within the rules of a strongly optimal solution, we have in this case a very simple certificate. The MPA party is entitled globally 8 seats. However, it should receive at least 1 seat in Campania 1 (otherwise it would incur the higher error 1.00179). Similarly it should receive at least 1 seat in Campania 2, Puglia and Calabria respectively. For the same reason it has to get at least 3 seats in Sicily. This makes already 7 seats. Therefore it is impossible to have two seats in Sicily 1.

As a further example suppose that the PDL party is complaining about the 9 seats in Piemonte 2 . Having a quota of 9.59563 the party claims 10 seats. The individual certificate for the pair (Piemonte 2, PDL) is given by the subsets:

$$
I=\{\text { Sicily } 1\}, \quad J=\{\text { PDL }, \text { MPA }\}
$$

from which we compute the $\alpha$ (on the left) and $\beta$ (on the right) values as in the Table 14, from which we get the contradiction

$$
\sum_{j \in J} p_{j}-\bar{\alpha}=280-266=14<\sum_{i \in I} r_{i}-\bar{\beta}=26-11=15
$$




\section{Conclusions}

In the design of sound electoral systems, some conflict may occur between accuracy and transparency. Indeed electoral problems like the biproportional apportionment one are not amenable to simple-minded solution, and when this is done several anomalies and paradoxes are likely to occur. On the other hand correct procedures may turn out to be too complex to be written down in an electoral law.

In this paper we have proposed a possible third way to get out of this dilemma. The key idea is to distinguish between the two stages of producing the solution and checking its validity. This idea is common in theoretical computer science, e.g. in the definition of the class NP. Luckily, for the particular min-max solution generated by the parametric max flow algorithm [16], this distinction is possible and a certificate can be produced on the basis of the Max Flow-Min Cut theorem. Obtaining such a certificate this way is not the task of the Verifier. However, it is nice to observe that its computation has low complexity.

While we have described a certificate with reference to biproportional apportionment and a particular error measure, similar ideas may have potential scope with different error measures and also in other contexts within the area, especially when writability is an important issue.

In conclusion, the adoption of a certificate of guarantee in presence of complex electoral rules which cannot be easily replicated by the layman, may result in better quality electoral systems without prejudice of transparency.

\section{References}

[1] R.K. Ahuja, T.L. Magnanti and J.B. Orlin: Network flows. Theory, algorithms and applications. Prentice Hall, New Jersey (1993).

[2] M.L. Balinski: Le Suffrage Universel Inachevè. Belin, Paris (2004).

[3] M. Balinski and G. Demange: An axiomatic approach to proportionality between matrices, Mathematics of Operations Research, 14, 700-719 (1989).

[4] M. Balinski and G. Demange: Algorithms for proportional matrices in reals and integers, Mathematical Programming, 45, 193-210 (1989).

[5] M.L. Balinski and V. Ramírez: Mexican Electoral Law: 1996 version, Electoral Studies, 16, 329-349 (1997).

[6] M. Balinski and H.P. Young: Fair Representation-Meeting the Ideal of One Man, One Vote. Second Edition. Brookings Institution Press, Washington, DC (2001).

[7] N. Gaffke and F. Pukelsheim: Divisor methods for proportional representation systems: An optimization approach to vector and matrix apportionment problems, Mathematical Social Sciences, 56, 166-184 (2008).

[8] N. Gaffke and F. Pukelsheim: Vector and matrix apportionment problems and separable convex integer optimization, Mathematical Methods of Operations Research, 67, 133-159 (2008).

[9] S. Maier and F. Pukelsheim: Bazi: A Free Computer Program for Proportional Representation Apportionment. Preprint Nr. 042/2007. Institut für Mathematik, Universität Augsburg, 2007. Internet: www.opus-bayern.de/uni-augsburg/volltexte/2007/711/ (2007).

[10] D. Gale: A theorem on flows in networks, Pacific Journal of Mathematics, 7, 1073-1082 (1957).

[11] Pennisi A.: The Italian bug: a flawed procedure for bi-proportional seat allocation, in: B. Simeone, F. Pukelsheim (eds), Mathematics and democracy: Recent advances in voting systems and collective choice, pp. 151-166, Berlin, Springer (2006). 
[12] Pennisi A., F. Ricca, B. Simeone: Malfunzionamenti dell'allocazione biproporzionale di seggi nella riforma elettorale italiana. Dipartimento di Statistica, Probabilità e Statistiche Applicate, Università La Sapienza, Roma, Serie A - Ricerche, n. 21 (2005).

[13] Pennisi A., F. Ricca, B. Simeone: Legge elettorale con paradosso. La Voce, 11 Novembre (2005).

[14] Pennisi A., F. Ricca, B. Simeone: Bachi e buchi della legge elettorale italiana nell'allocazione biproporzionale di seggi, Sociologia e Ricerca Sociale, 79, 55-76 (2006).

[15] F. Pukelsheim: BAZI: A Java program for proportional representation. Oberwolfach Reports 1, 735737, www .uni-augsburg.de/bazi (2004).

[16] P. Serafini and B. Simeone: Parametric maximum flow methods for minimax approximation of target quotas in biproportional apportionment, Networks, forthcoming.

[17] B. Simeone, F. Pukelsheim (eds.): Mathematics and Democracy. Recent Advances in Voting Systems and Collective Choice. Studies in Choice and Welfare, Springer (2006). 
Table 11: Target quotas

\begin{tabular}{|l|r|r|r|r|r|r|c|}
\hline & PDL & LN & MPA & PD & IDV & SVP & UDC \\
\hline Piemonte 1 & 9.470 & 2.530 & 0.000 & 9.450 & 1.550 & 0.000 & 1.283 \\
\hline Piemonte 2 & 9.596 & 4.404 & 0.000 & 6.150 & 0.850 & 0.000 & 1.194 \\
\hline Lombardia 1 & 16.580 & 7.421 & 0.000 & 13.080 & 1.920 & 0.000 & 1.428 \\
\hline Lombardia 2 & 15.350 & 13.650 & 0.000 & 10.390 & 1.613 & 0.000 & 2.064 \\
\hline Lombardia 3 & 5.829 & 3.171 & 0.000 & 4.534 & 0.466 & 0.000 & 0.689 \\
\hline Trentino-Alto Adige & 2.758 & 1.242 & 0.000 & 2.642 & 0.358 & 2.644 & 0.460 \\
\hline Veneto 1 & 9.312 & 9.688 & 0.000 & 7.806 & 1.194 & 0.000 & 1.754 \\
\hline Veneto 2 & 6.265 & 5.736 & 0.000 & 5.955 & 1.045 & 0.000 & 1.013 \\
\hline Friuli-Venezia Giulia & 5.089 & 1.911 & 0.000 & 4.398 & 0.602 & 0.000 & 0.815 \\
\hline Liguria & 7.588 & 1.412 & 0.000 & 6.189 & 0.811 & 0.000 & 0.669 \\
\hline Emilia-Romagna & 14.940 & 4.058 & 0.000 & 20.140 & 1.862 & 0.000 & 1.918 \\
\hline Toscana & 15.030 & 0.969 & 0.000 & 18.610 & 1.390 & 0.000 & 1.700 \\
\hline Umbria & 3.816 & 0.184 & 0.000 & 4.683 & 0.317 & 0.000 & 0.437 \\
\hline Marche & 6.585 & 0.415 & 0.000 & 7.216 & 0.784 & 0.000 & 1.035 \\
\hline Lazio 1 & 19.870 & 0.000 & 0.131 & 16.120 & 1.881 & 0.000 & 1.800 \\
\hline Lazio2 & 8.927 & 0.000 & 0.073 & 4.558 & 0.442 & 0.000 & 0.940 \\
\hline Abruzzo & 6.738 & 0.000 & 0.262 & 4.961 & 1.039 & 0.000 & 0.858 \\
\hline Molise & 1.744 & 0.000 & 0.256 & 0.393 & 0.607 & 0.000 & 0.176 \\
\hline Campania 1 & 18.000 & 0.000 & 1.002 & 10.280 & 1.720 & 0.000 & 1.836 \\
\hline Campania 2 & 16.320 & 0.000 & 0.681 & 8.646 & 1.354 & 0.000 & 2.207 \\
\hline Puglia & 23.100 & 0.000 & 0.900 & 13.950 & 2.048 & 0.000 & 3.581 \\
\hline Basilicata & 2.938 & 0.000 & 0.062 & 2.601 & 0.399 & 0.000 & 0.438 \\
\hline Calabria & 11.290 & 0.000 & 0.707 & 7.202 & 0.798 & 0.000 & 1.918 \\
\hline Sicilia 1 & 13.370 & 0.000 & 1.626 & 6.953 & 1.048 & 0.000 & 2.968 \\
\hline Sicilia 2 & 14.980 & 0.000 & 3.025 & 7.159 & 0.841 & 0.000 & 2.114 \\
\hline Sardegna & 8.868 & 0.000 & 0.132 & 7.209 & 0.791 & 0.000 & 1.059 \\
\hline & & & & & & & \\
\hline
\end{tabular}


Table 12: Apportionment minimizing the maximum absolute error

\begin{tabular}{|c|c|c|c|c|c|c|c|}
\hline & PDL & $\mathrm{LN}$ & MPA & $\mathrm{PD}$ & IDV & SVP & UDC \\
\hline Piemonte 1 & 9 & 3 & 0 & 9 & 2 & 0 & 1 \\
\hline Piemonte 2 & 9 & 5 & 0 & 6 & 1 & 0 & 1 \\
\hline Lombardia 1 & 16 & 8 & 0 & 13 & 2 & 0 & 1 \\
\hline Lombardia 2 & 15 & 14 & 0 & 10 & 2 & 0 & 2 \\
\hline Lombardia 3 & 6 & 3 & 0 & 5 & 0 & 0 & 1 \\
\hline Trentino-Alto Adige & 3 & 1 & 0 & 3 & 0 & 2 & 1 \\
\hline Veneto 1 & 9 & 10 & 0 & 8 & 1 & 0 & 1 \\
\hline Veneto 2 & 6 & 6 & 0 & 6 & 1 & 0 & 1 \\
\hline Friuli-Venezia Giulia & 5 & 2 & 0 & 4 & 1 & 0 & 1 \\
\hline Liguria & 7 & 2 & 0 & 6 & 1 & 0 & 1 \\
\hline Emilia-Romagna & 15 & 4 & 0 & 20 & 2 & 0 & 2 \\
\hline Toscana & 15 & 1 & 0 & 19 & 1 & 0 & 2 \\
\hline Umbria & 4 & 0 & 0 & 5 & 0 & 0 & 0 \\
\hline Marche & 6 & 1 & 0 & 7 & 1 & 0 & 1 \\
\hline Lazio 1 & 20 & 0 & 0 & 16 & 2 & 0 & 2 \\
\hline Lazio 2 & 9 & 0 & 0 & 5 & 0 & 0 & 1 \\
\hline Abruzzo & 7 & 0 & 0 & 5 & 1 & 0 & 1 \\
\hline Molise & 2 & 0 & 0 & 0 & 1 & 0 & 0 \\
\hline Campania 1 & 18 & 0 & 1 & 10 & 2 & 0 & 2 \\
\hline Campania 2 & 16 & 0 & 1 & 9 & 1 & 0 & 2 \\
\hline Puglia & 23 & 0 & 1 & 14 & 2 & 0 & 4 \\
\hline Basilicata & 3 & 0 & 0 & 3 & 0 & 0 & 0 \\
\hline Calabria & 11 & 0 & 1 & 7 & 1 & 0 & 2 \\
\hline Sicilia 1 & 14 & 0 & 1 & 7 & 1 & 0 & 3 \\
\hline Sicilia 2 & 15 & 0 & 3 & 7 & 1 & 0 & 2 \\
\hline Sardegna & 9 & 0 & 0 & 7 & 1 & 0 & 1 \\
\hline
\end{tabular}


Table 13:

\begin{tabular}{|r|r|r|r|r|r|r|}
\hline & PDL & MPA & PD & IDV & SVP & UDC \\
\hline Pi2 & 9 & 0 & 6 & 1 & 0 & 1 \\
\hline Lo1 & 16 & 0 & 13 & 2 & 0 & 1 \\
\hline Ve1 & 9 & 0 & 8 & 1 & 0 & 2 \\
\hline Ve2 & 6 & 0 & 6 & 1 & 0 & 1 \\
\hline ERm & 15 & 0 & 20 & 2 & 0 & 2 \\
\hline Mar & 6 & 0 & 7 & 1 & 0 & 1 \\
\hline La1 & 20 & 0 & 16 & 2 & 0 & 2 \\
\hline Si2 & 15 & 3 & 7 & 1 & 0 & 2 \\
\hline Sar & 9 & 0 & 7 & 1 & 0 & 1 \\
\hline
\end{tabular}

$\alpha_{i j}$

\begin{tabular}{|c|r|}
\hline & LN \\
\hline Pi1 & 3 \\
\hline Lo2 & 14 \\
\hline Lo3 & 3 \\
\hline TrA & 1 \\
\hline FVG & 2 \\
\hline Lig & 2 \\
\hline Tos & 1 \\
\hline Umb & 0 \\
\hline La2 & 0 \\
\hline Abr & 0 \\
\hline Mol & 0 \\
\hline Cam1 & 0 \\
\hline Cam2 & 0 \\
\hline Pug & 0 \\
\hline Bas & 0 \\
\hline Cal & 0 \\
\hline Si1 & 0 \\
\hline
\end{tabular}


Table 14:

\begin{tabular}{|r|r|r|}
\hline & PDL & MPA \\
\hline Pi1 & 9 & 0 \\
\hline Pi2 & 10 & 0 \\
\hline Lo1 & 16 & 0 \\
\hline Lo2 & 15 & 0 \\
\hline Lo3 & 6 & 0 \\
\hline TrA & 3 & 0 \\
\hline Ve1 & 9 & 0 \\
\hline Ve2 & 6 & 0 \\
\hline FVG & 5 & 0 \\
\hline Lig & 7 & 0 \\
\hline ERm & 15 & 0 \\
\hline Tos & 15 & 0 \\
\hline Umb & 4 & 0 \\
\hline Mar & 6 & 0 \\
\hline La1 & 20 & 0 \\
\hline La2 & 9 & 0 \\
\hline Abr & 7 & 0 \\
\hline Mol & 2 & 0 \\
\hline Cam1 & 18 & 1 \\
\hline Cam2 & 16 & 1 \\
\hline Pug & 23 & 1 \\
\hline Bas & 3 & 0 \\
\hline Cal & 11 & 1 \\
\hline Si2 & 15 & 3 \\
\hline Sar & 9 & 0 \\
\hline & $\alpha_{i j}$ & \\
\hline
\end{tabular}

\begin{tabular}{|r|r|r|r|r|r|}
\hline & LN & PD & IDV & SVP & UDC \\
\hline Si1 & 0 & 7 & 1 & 0 & 3 \\
\hline
\end{tabular}

$\beta_{i j}$ 